\title{
Heavy-quark correlations in deep inelastic scattering
}

\author{
J. Smith ${ }^{\mathrm{a} *}$ and B.W. Harris ${ }^{\mathrm{b}} \dagger$ \\ ${ }^{a}$ Institute for Theoretical Physics, State University of New York at Stony Brook, New York, \\ 11794-3840, USA \\ ${ }^{\text {b} P h y s i c s ~ D e p a r t m e n t, ~ F l o r i d a ~ S t a t e ~ U n i v e r s i t y, ~ T a l l a h a s s e e, ~ F l o r i d a, ~ 32306-3016, ~ U S A ~}$
}

We discuss some results for heavy quark correlations in next-to-leading order in deep inelastic electroproduction.

\section{INTRODUCTION}

Order $\alpha_{s}$ QCD corrections to structure functions containing heavy quarks and to single heavy quark inclusive distributions in deep-inelastic electroproduction (i.e., $\gamma^{*}(q)+P(p) \rightarrow Q\left(p_{1}\right)+$ $X$ where $X$ stands for any final hadronic state allowed by quantum-number conservation and $P(p)$ is a proton of momentum $p$ ) were recently published in [1] and [2], respectively. By combining the next-to-leading order (NLO) heavy quark structure functions with the corresponding lightquark structure functions [3], the heavy quark content of the nucleon has been studied as a function of $Q^{2}=-q^{2}$ and $x=Q^{2} / 2 p \cdot q$ 顿. Event rates for charm production integrated over bins in $x$ and $Q^{2}$ relevant to HERA data have been calculated in $\mid 5$.

To further the study of deep-inelastic electroproduction of heavy quarks we have recalculated the virtual-photon-parton cross sections of [1] in an exclusive fashion [6]. This enables us to study the single and double differential distributions and correlations among all outgoing particles in the virtual-photon induced reaction $\gamma^{*}+P \rightarrow$ $Q+\bar{Q}+X$ with $X=0$ or 1 jet and to easily incorporate experimental cuts. By examining distributions and correlations that are trivial at lowest order (for example, the azimuthal angle between the heavy quark and the heavy antiquark) one directly tests the hard scattering cross section that is predicted by perturbative QCD and factorization. Therefore our results should give a clean

\footnotetext{
*Partially supported under the contract NSF-09888.

${ }^{\dagger}$ Supported under the contract DOE-FG05-87ER40319.
}

test of NLO perturbative QCD. Nevertheless we remind the reader that this is a fixed order perturbative calculation and suffers from the same problems as all NLO calculations. There are regions in phase space where it will break down. For example, in the above mentioned azimuthal angle distribution, if one looks at the prediction too near the back-to-back configuration there will be an extra enhancement of the cross section due to multiple soft gluon emission which is not included in our NLO result.

In this short report we discuss some details of the calculation and present interesting distributions. The transverse and longitudinal photon components are treated separately and the latest CTEQ3 parton densities [7], consistent with the newly released HERA data [8], are used in the kinematic regime appropriate for production of charm quarks at HERA. We make our predictions at fixed values of $Q^{2}\left(\geq 8.5(\mathrm{GeV} / \mathrm{c})^{2}\right)$ and $x\left(\geq 4.2 \times 10^{-4}\right)$.

We stress that here we only consider extrinsic heavy quark production, involving Bethe-Heitler and Compton production from massless partons. For a discussion of intrinsic production, where the heavy quark is considered to be part of the proton's wavefunction, see Brodsky et al. [9]. A variable flavor scheme which joins the extrinsic heavy flavor production picture at $\mu_{\text {phys }} \ll m$ with a light mass parton density picture at $\mu_{\text {phys }} \gg m$ has been discussed by Aivazis et al. [10]. By comparing the fixed flavor scheme calculation of |1] with the variable flavor number scheme of 10 it is concluded in 11] that the former yields the most stable and reliable results for $F_{2}\left(x, Q^{2}, m^{2}\right)$ 
in the threshold region (i.e., $Q^{2} \leq 10 m^{2}$ where $m$ is the mass of the heavy quark). The contribution by W.L. van Neerven to these Proceedings reviews the progress towards implementing the variable flavour number scheme in NLO 12].

In our exclusive computation we use the subtraction method which is based on the replacement of divergent (collinear or soft) terms in the squared matrix elements by generalized plus distributions. This allows us to isolate the soft and collinear poles within the framework of dimensional regularization without calculating all the phase space integrals in a spacetime dimension $n \neq 4$ as usually required in a traditional single particle inclusive computation. In this method the expressions for the squared matrix elements in the collinear limit appear in a factorized form, where poles in $n-4$ multiply splitting functions and lower order squared matrix elements. The cancellation of collinear singularities is then performed using the factorization theorem [13]. The expressions for the squared matrix elements in the soft limit appear in a factorized form where poles in $n-4$ multiply lower order squared matrix elements. The cancellation of soft singularities takes place upon adding the contributions from the renormalized virtual diagrams. Since the final result is in four-dimensional space time, we can compute all relevant phase space integrations using standard Monte Carlo integration techniques [14] and produce histograms for exclusive, semiinclusive, or inclusive quantities related to any of the outgoing particles. We can also incorporate any reasonable set of experimental cuts. Our computer code has no small phase space slice parameters and the parameters defining the generalized plus distributions may be tuned to give fast numerical convergence, which is an advantage of using this subtraction method.

\section{The $\gamma^{*} q$ channel}

Analysis of the partonic reaction

$\gamma^{*}(q)+q\left(k_{1}\right) \rightarrow q\left(k_{2}\right)+Q\left(p_{1}\right)+\bar{Q}\left(p_{2}\right)$,

does not involve soft or virtual contributions so we can use it to most simply explain the method. One particular graph out of the order $e g^{2}$ set is

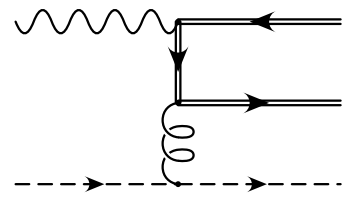

Figure 1. One order $e g^{2}$ diagram contributing to the amplitude for the reaction (1). Additional graphs are obtained by reversing the arrows on the light-quark lines (dashed).

shown in fig.1. We project this set of graphs on a particular photon polarization state $i$ and square the amplitude. The answer is written as $C_{i, q} M_{i}^{q}(3)$ where the coefficient absorbs overall factors. Now we write the photon-parton cross section as

$d \sigma_{i, q}^{(3)}=C_{i, q} M_{i}^{q}(3) d \Gamma_{3}$.

where the 3 indicates a three-body final state. To proceed we need some notation for the variables $s=2 k_{1} \cdot q, t_{1}=t-m^{2}=-2 k_{1} \cdot p_{2}, u_{1}^{\prime}=u_{1}-q^{2}=$ $2 q \cdot p_{2}, t^{\prime}=-2 k_{1} \cdot k_{2}, u^{\prime}=q^{2}-2 q \cdot k_{2}$ and $s_{5}=$ $\left(p_{1}+p_{2}\right)^{2}-q^{2}$. Then if we introduce $x=s_{5} / s$ and $\cos ^{-1} y=\vec{q} \cdot \vec{k}_{2} /\left|\vec{q} \cdot \vec{k}_{2}\right|$ we can write $t^{\prime}=$ $-(1 / 2) s^{\prime}\left(s^{\prime} / s\right)(1-x)(1+y)$. This representation shows that $t^{\prime} \rightarrow 0$ as $y \rightarrow-1$. This $t^{\prime}$ divergence yields the collinear divergence in $M_{i}^{q}(3)$, which is due to the presence of the Feynman graph in fig.1. To handle this divergence we multiply and divide by $t^{\prime}$ so that

$d \sigma_{i, q}^{(3)}=C_{i, q} f_{i}^{q}\left(x, y, \theta_{1}, \theta_{2}\right) \overline{d \Gamma_{3}}$,

where

$f_{i}^{q}\left(x, y, \theta_{1}, \theta_{2}\right) \equiv t^{\prime} M_{i}^{q}(3)$,

is now finite as $y \rightarrow-1$ and

$\overline{d \Gamma_{3}} \equiv d \Gamma_{3} / t^{\prime}$.

is divergent. We next replace the factor

$(1+y)^{-1+\epsilon / 2}=(1 /(1+y))_{\omega}+\delta(1+y)(1 / \epsilon+\ln \omega),(6)$ 
in the ( $n$-dimensional) three-body phase space, where $0<\omega<2$. This has the effect of splitting the $n$-dimensional cross section into two divergent parts. However it lets us take the limit $\epsilon \rightarrow 0$ and get back to 4 -dimensions, with $\omega$ regularizing the collinear singularity. Replacing the divergent factor $(1+y)^{-1+\epsilon / 2}$ in $\overline{d \Gamma_{3}}$ one obtains the following decomposition: (for more details see [6])

$d \sigma_{i, q}^{(3)}=d \sigma_{i, q}^{(c-)}+d \sigma_{i, q}^{(f)}$,

with

$$
\begin{aligned}
d \sigma_{i, q}^{(c-)} & =-\frac{1}{\pi} C_{i, q} H N d \Gamma_{2}\left(s^{\prime}\right)^{\epsilon / 2}\left(\frac{s^{\prime}}{s}\right)^{\epsilon / 2} \\
& \times(1-x)^{\epsilon}(1-y)^{\epsilon / 2} \delta(1+y)\left(\frac{2}{\epsilon}+\ln \omega\right) \\
& \times d y \sin ^{\epsilon} \theta_{2} d \theta_{2} f_{i}^{q}\left(x, y, \theta_{1}, \theta_{2}\right),
\end{aligned}
$$

and

$$
\begin{aligned}
d \sigma_{i, q}^{(f)} & =-\frac{1}{\pi} C_{i, q} H N d \Gamma_{2}\left(s^{\prime}\right)^{\epsilon / 2}\left(\frac{s^{\prime}}{s}\right)^{\epsilon / 2} \\
& \times(1-x)^{\epsilon}(1-y)^{\epsilon / 2}\left(\frac{1}{1+y}\right)_{\omega} \\
& \times d y \sin ^{\epsilon} \theta_{2} d \theta_{2} f_{i}^{q}\left(x, y, \theta_{1}, \theta_{2}\right) .
\end{aligned}
$$

The function $f$ satisfies

$f_{i}^{q}\left(x,-1, \theta_{1}, \theta_{2}\right)=f_{i}^{q}\left(x, \theta_{1}\right)+\tilde{f}_{i}^{q}\left(x, \theta_{1}, \theta_{2}\right)$,

with

$$
\int_{0}^{\pi} \tilde{f}_{i}^{q}\left(x, \theta_{1}, \theta_{2}\right) \sin ^{\epsilon} \theta_{2} d \theta_{2}=0 .
$$

For reaction (1) we find that

$$
\begin{aligned}
f_{i}^{q}\left(x, \theta_{1}\right) & =-128 \pi^{2} \mu^{-2 \epsilon} \alpha_{s}^{2} e^{2} e_{H}^{2} N C_{F} \\
& \times\left[\frac{1+(1-x)^{2}+\epsilon x^{2} / 2}{x^{2}}\right] \\
& \times(1+\epsilon / 2)^{-1} B_{i, Q E D}\left(x k_{1}\right) .
\end{aligned}
$$

where $B_{i, Q E D}$ it the Born amplitude with $k_{1}$ replaced by $x k_{1}$. The factor in the square brackets is the Altarelli-Parisi splitting function in $n$ dimensions so we can define new (collinear free) cross sections

$d \hat{\sigma}_{i, q}^{(3)}=d \hat{\sigma}_{i, q}^{(c-)}+d \sigma_{i, q}^{(f)}$,

with

$d \hat{\sigma}_{i, q}^{(c-)}=8 C_{i, q} \alpha_{s}^{2} e^{2} e_{H}^{2} N C_{F} B_{i, Q E D}\left(x k_{1}\right) d \Gamma_{2}$

$$
\begin{aligned}
\times & \left\{1+\frac{1+(1-x)^{2}}{x^{2}}\left[\ln \frac{s^{\prime}}{\mu^{2}}+\ln \frac{s^{\prime}}{s}\right.\right. \\
& \left.\left.+\ln \frac{\omega}{2}+2 \ln (1-x)\right]\right\} \\
d \sigma_{i, q}^{(f)}= & -\left(\frac{1}{16 \pi^{2}}\right)^{2} C_{i, q} \beta_{5} f_{i}^{q}\left(x, y, \theta_{1}, \theta_{2}\right) \\
\times & \left(\frac{1}{1+y}\right)_{\omega} d x d y \sin \theta_{1} d \theta_{1} d \theta_{2} .
\end{aligned}
$$

The finite functions $f_{i}^{q}\left(x, y, \theta_{1}, \theta_{2}\right)$ are available in [6].

As the quark channel only contains collinear singularities, we also use it to illustrate how the generalized plus distributions are implemented numerically and how the $\omega$ dependence disappears in the sum (13). To this end consider

$$
\begin{aligned}
d \sigma_{i, q}^{(f)} & \sim \int_{-1}^{1} d y f(y)\left(\frac{1}{1+y}\right)_{\omega} \\
= & \int_{-1}^{-1+\omega} d y f(y)\left(\frac{1}{1+y}\right)_{\omega} \\
& +\int_{-1+\omega}^{1} d y f(y)\left(\frac{1}{1+y}\right)_{\omega} \\
=\quad & \int_{-1}^{-1+\omega} d y \frac{f(y)-f(-1)}{1+y} \\
& +\int_{-1+\omega}^{1} d y \frac{f(y)}{1+y} \\
=\quad & \int_{-1}^{1} d y \frac{f(y)}{1+y}-\int_{-1}^{-1+\omega} d y \frac{f(-1)}{1+y}
\end{aligned}
$$

where we have suppressed all indices and arguments of $f_{i}^{q}\left(x, y, \theta_{1}, \theta_{2}\right)$ other than $y$. In the bottom line we see that the infinity encountered at the lower integration limit $y=-1$ is cancelled in the sum of the two integrals. In practice one can only reasonably take the lower limit to be $-1+\delta$ where $\delta \sim 10^{-7}$ in double precision FORTRAN before round off errors enter. None the less, the final results are stable with respect to the variation of $\delta$ in the range $10^{-5}$ to $10^{-7}$. The upper limit of the second integral gives a contribution $f(-1) \ln \omega$ which cancels against the $\ln \omega$ term in $d \hat{\sigma}_{i, q}^{(c-)}$. The first integral in the bottom line is commonly called the "event" and has a positive definite weight. The second integral plus the factorized collinear contribution is commonly called 
the "counter-event" and may have either positive or negative weight.

The implementation of this procedure for the gluon channel is similar but more complicated due to the presence of both soft and collinear divergences.

\section{Results}

Recalling that the probability density is related to the momentum density via $f_{i / H}\left(\xi, \mu_{f}^{2}\right)=$ $\xi \phi_{i / H}\left(\xi, \mu_{f}^{2}\right)$ we write the hadronic cross section as

$d \sigma_{\gamma^{*} H}(p)=\sum_{i} \int_{0}^{1} \frac{d \xi}{\xi} d \hat{\sigma}_{i}(\xi p) f_{i / H}\left(\xi, \mu_{f}^{2}\right)$.

We now specialize to the case where $H$ is a proton, as in the case of HERA. Using the relations

$F_{k}=\frac{Q^{2}}{4 \pi^{2} \alpha} \sigma_{k}$,

where $k=2, L$ with $\sigma_{2}=\sigma_{G}+3 \sigma_{L} / 2$, and relations for the scaling functions, we find

$$
\begin{aligned}
& F_{k}\left(x, Q^{2}, m^{2}\right)=\frac{Q^{2} \alpha_{s}\left(\mu^{2}\right)}{4 \pi^{2} m^{2}} \int_{\xi_{\min }}^{1} \frac{d \xi}{\xi} \\
& \times e_{H}^{2} f_{g / P}\left(\xi, \mu^{2}\right) c_{k, g}^{(0)} \\
& +\frac{Q^{2} \alpha_{s}^{2}\left(\mu^{2}\right)}{\pi m^{2}} \int_{\xi_{\min }}^{1} \frac{d \xi}{\xi} \\
& \times\left\{e_{H}^{2} f_{g / P}\left(\xi, \mu^{2}\right)\left(c_{k, g}^{(1)}+\bar{c}_{k, g}^{(1)} \ln \frac{\mu^{2}}{m^{2}}\right)\right. \\
& +\sum_{i=q, \bar{q}} f_{i / P}\left(\xi, \mu^{2}\right)\left[e_{H}^{2}\left(c_{k, i}^{(1)}+\bar{c}_{k, i}^{(1)} \ln \frac{\mu^{2}}{m^{2}}\right)\right. \\
& \left.\left.+e_{i}^{2} d_{k, i}^{(1)}+e_{i} e_{H} o_{k, i}^{(1)}\right]\right\},
\end{aligned}
$$

where $k=2, L$. We have set $\mu_{f}=\mu$ and shown the $\mu^{2}$ dependence of the running coupling $\alpha_{s}$ explicitly. The lower boundary on the integration is given by $\xi_{\min }=x\left(4 m^{2}+Q^{2}\right) / Q^{2}$. This formula yields the standard heavy quark structure functions $F_{2}\left(x, Q^{2}, m^{2}\right)$ and $F_{L}\left(x, Q^{2}, m^{2}\right)$ for electron proton scattering, and we will present results as differentials of these functions. From the formalism described in the previous section we are

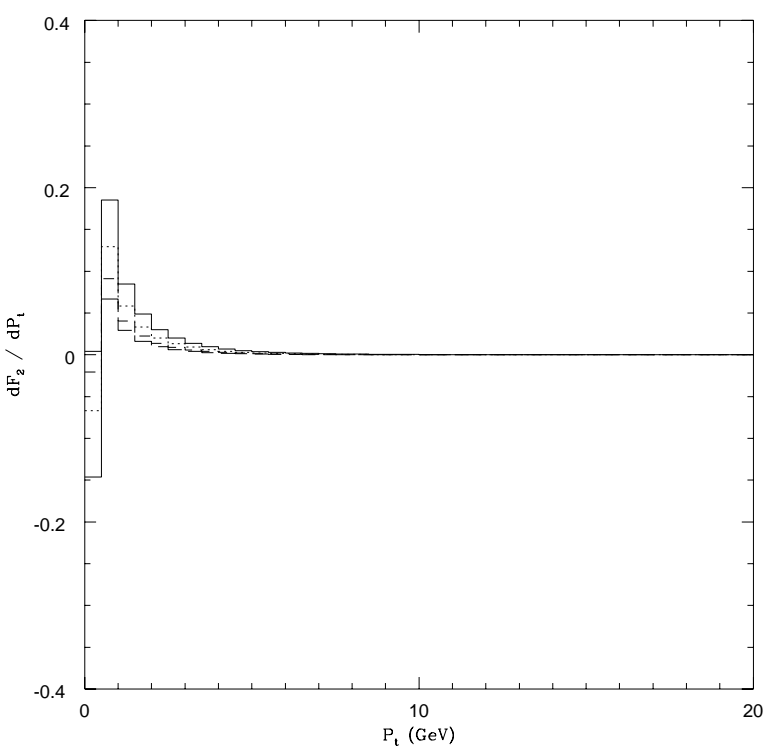

Figure 2. The distributions $d F_{2}\left(P_{t}\right) / d P_{t}$ for charm-anticharm pair production at fixed $Q^{2}=$ $12(\mathrm{GeV} / \mathrm{c})^{2}$ with $x=4.2 \times 10^{-4}$ (solid line), $8.5 \times 10^{-4}$ (dotted line), $1.6 \times 10^{-3}$ (short dashed line) and $2.7 \times 10^{-3}$ (long dashed line).

left with events of positive weight and counterevents of either positive or negative weight. Our program outputs the final state four vectors of the event and counter-event together with the corresponding weight. We histogram these into bins to produce differential distributions.

We start by considering the production of charm quarks. We use $m=m_{c}=1.5 \mathrm{GeV} / \mathrm{c}^{2}$ and simply choose the factorization (renormalization) scale as $\mu^{2}=Q^{2}+4\left(m_{c}^{2}+\left(P_{t}^{c}+P_{t}^{\bar{c}}\right)^{2} / 4\right)$. Note that there are many possible choices of scale as we have all components of the final four vectors. Aside from the $P_{t}$ dependence, this choice reduces to $\mu^{2}=Q^{2}$ for electroproduction of massless quarks and $\mu^{2}=4 m_{c}^{2}$ for the photoproduction of charm quarks. We introduce a $P_{t}$ dependence by adding in the average of the magnitude of the transverse momenta of the heavy quark and heavy antiquark. As mentioned earlier we use the CTEQ3M parton densities [7] in the $\overline{\mathrm{MS}}$ scheme 


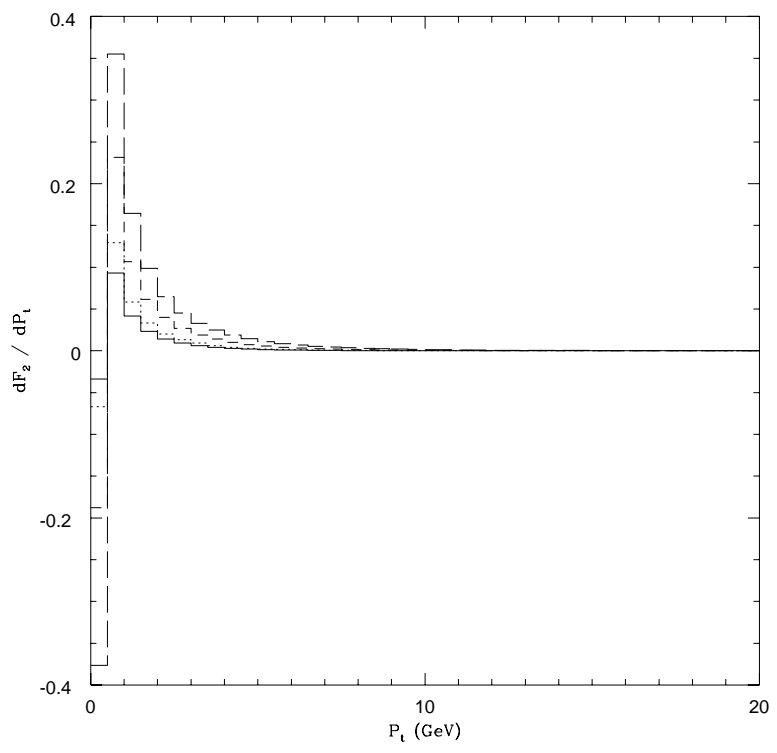

Figure 3. The distributions $d F_{2}\left(P_{t}\right) / d P_{t}$ for charm-anticharm pair production at fixed $x=$ $8.5 \times 10^{-4}$ and $Q^{2}=8.5$ (solid line), 12 (dotted line), 25 (short dashed line), 50 (long dashed line) all in units of $(\mathrm{GeV} / \mathrm{c})^{2}$.

and the two loop $\alpha_{s}$ with $\Lambda_{4}=0.239 \mathrm{GeV}$.

The first distribution we present basically measures the transverse momentum of the additional jet which recoils against the heavy quark pair. The $P_{t}$ distribution of the charm-anticharm pair is shown in fig. 2 where we plot $d F_{2}\left(x, Q^{2}, m_{c}^{2}, P_{t}\right) / d P_{t}$ as a function of $P_{t}$. The histograms are presented at fixed $Q^{2}=12$ $(\mathrm{GeV} / c)^{2}$ for $x$ values of $4.2 \times 10^{-4}, 8.5 \times 10^{-4}$, $1.6 \times 10^{-3}$ and $2.7 \times 10^{-3}$ respectively. One sees that the $P_{t}$ distribution peaks at small $P_{t}$ and has a negative contribution in the lowest bin. This is a region where the dominant contribution is from counter-events so the weights can be negative. The results of this calculation require missing contributions from even higher order perturbation theory before this bin will have a positive weight. The depth of the negative bins is a function of $x, Q^{2}$, and the choice of scale. Note that at larger $P_{t}$ the structure function is domi- nated by the contribution from the square of the bremsstrahlung graphs so the weights are positive. Figure 3 shows the corresponding results for fixed $x=8.5 \times 10^{-4}$ plotted for the $Q^{2}$ values of $8.5(\mathrm{GeV} / c)^{2}, 12(\mathrm{GeV} / c)^{2}, 25(\mathrm{GeV} / c)^{2}$ and $50(\mathrm{GeV} / c)^{2}$ respectively. The distributions peak near small $P_{t}$ and are either small or negative in the first bin. The histograms with $Q^{2}=$ $12(\mathrm{GeV} / c)^{2}$ and $x=8.5 \times 10^{-4}$ (the dotted line) are the same in figs. 2 and 3 . We have also used the same scales on the axes so one can easily see that there is a greater change if we fix $x$ and vary $Q^{2}$ than if we fix $Q^{2}$ and vary $x$. We will continue to use the same scale for all the pairs of later plots to simplify the comparison between them.

We now turn to the distributions in the azimuthal angle between the outgoing charm quark and charm antiquark which we denote as $\Delta \phi$. This is the angle between the $\mathbf{P}_{t}$ vectors of the heavy quark-antiquark in the c. m. frame of the virtual-photon-hadron system. Since we integrate over the azimuthal angle between the plane containing the incoming and outgoing leptons and the plane defined by the incoming parton and outgoing heavy quark (to limit our discussion to $F_{2}$ and $F_{L}$ ) we can only plot relative azimuthal correlations. In the Born approximation this distribution is a delta function at $\pi$, as their four momenta must balance. Due to the radiation of the additional light mass parton, the distribution has a tail extending below $\pi$ and has a valley at $\pi$. The distributions become negative in the highest bins. This negative region is a general feature of all exclusive calculations. Figure 4 shows results for $d F_{2}\left(x, Q^{2}, m_{c}^{2}, \Delta \phi\right) / d(\Delta \phi)$ at the same values of fixed $Q^{2}$ and variable $x$ as chosen previously in fig. 2, while fig. 5 shows the results for fixed $x$ and variable $Q^{2}$ as chosen previously in fig. 3 . Note again that the dotted histograms are the same in figs. 4 and 5 , and there is more variation for fixed $x$ and changing $Q^{2}$ than for fixed $Q^{2}$ and changing $x$.

In this report we have outlined the NLO calculation of the virtual-photon-parton (Wilson) coefficient functions in the fully differential production of heavy quarks plus one jet. More correlations and checks are given in [6]. The computer program we have written for the exclusive cal- 


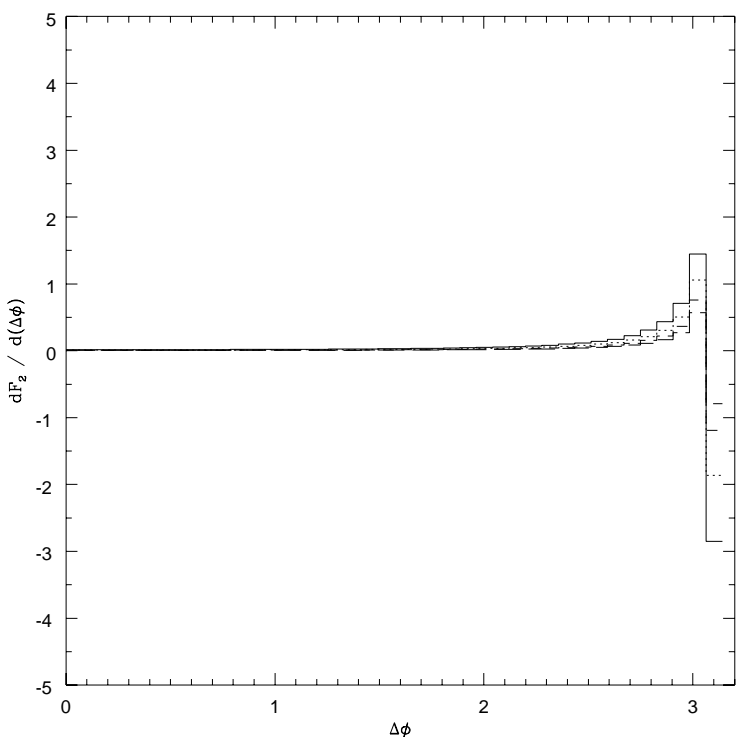

Figure 4. The distributions $d F_{2}(\Delta \phi) / d(\Delta \phi)$ for charm-anticharm pair production at the $x$ and $Q^{2}$ values given in Figure 2.

culation has the advantage that the four vectors of the heavy quark, heavy-antiquark and/or one additional light parton jet are produced for each event and can be subjected to experimental cuts. We have not done this in any of the plots shown here but the computer program is available and can be easily modified to incorporate acceptances of the detectors at HERA. The program has been used to reanalyse the EMC data on charm production in [17].

\section{Conclusion}

In this report we have presented some plots which depend on information from the four vector of the additional jet. We showed the distributions in the transverse-momentum $\left(P_{t}\right)$ of the heavy quark antiquark pair and in the azimuthal angle $(\Delta \phi)$ between the $\mathbf{P}_{t}$ vectors of the heavy quark and heavy antiquark. All quantities were predicted in the c. $m$. frame of the photon-proton system after integration over the azimuthal angle between the plane containing the incoming and out-

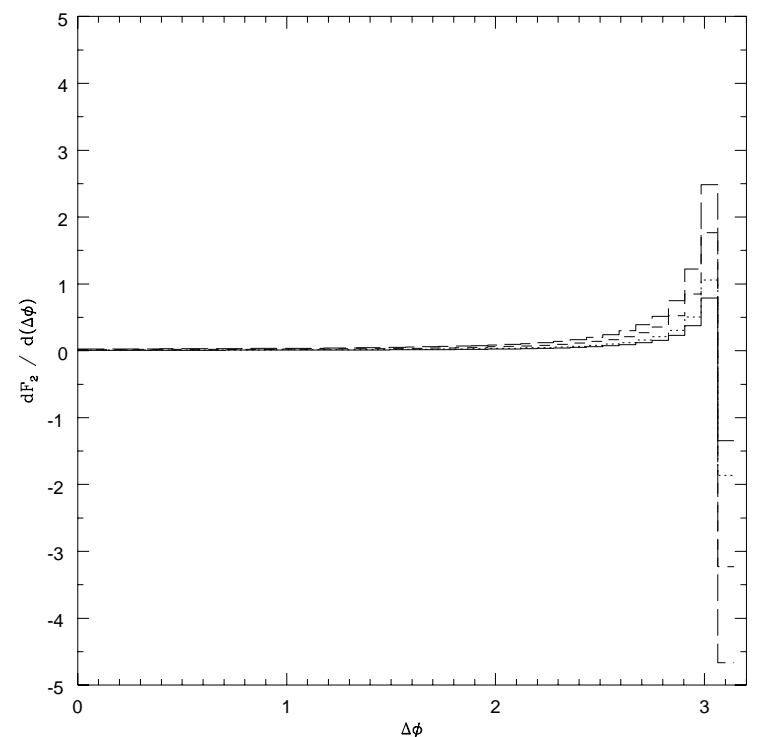

Figure 5. The same distributions at the $x$ and $Q^{2}$ values given in Figure 3.

going lepton and the plane containing the incoming proton and outgoing heavy quark. The results were presented as distributions in $F_{2}\left(x, Q^{2}, m^{2}\right)$ at specific points in $x, Q^{2}$ and $m^{2}=m_{c}^{2}$. None of these distributions can be reproduced by any $K$ factor multiplication as the corresponding Born distributions are proportional to delta-functions. In all cases the histograms of these distributions have negative bins. These are regions where the NLO calculation is not sufficient and a NNLO order calculation (or some form of resummation) should be made. A general statement about the magnitude of the NLO contribution compared to the $\mathrm{LO}$ one is difficult to make as the size and sign of the corrections may vary strongly between different regions of phase space. However, we see that all plots have larger $Q^{2}$ variation at fixed $x$ as comparied to varying $x$ at fixed $Q^{2}$. By varying the renormalization (factorization) scale we observed that the distributions presented here changed in normalization but not in shape. In our study we also calculated averages of various quantities and found the typical variation between cen- 
tral and extreme scale choices of 5 percent for charm production.

\section{REFERENCES}

1. E. Laenen, S. Riemersma, J. Smith and W. L. van Neerven, Nucl. Phys. B392 (1993) 162.

2. E. Laenen, S. Riemersma, J. Smith and W. L. van Neerven, Nucl. Phys. B392 (1993) 229.

3. E. B. Zijlstra and W. L. van Neerven, Nucl. Phys. B383, 525 (1992).

4. E. Laenen, S. Riemersma, J. Smith and W. L. van Neerven, Phys. Lett. B291 (1992) 325.

5. S. Riemersma, J. Smith and W. L. van Neerven, Phys. Lett. B347 (1995) 143.

6. B.W. Harris and J. Smith, Nucl. Phys. B452 (1995) 109.

7. H. L. Lai, J. Botts, J. Huston, J. G. Morfín, J. F. Owens, J. W. Qiu, W-K. Tung and H. Weerts, Phys. Rev. D51 (1995) 4763.

8. H1 Collaboration, I. Abt et al., Nucl. Phys. B407 (1993) 515; ZEUS Collaboration, M. Derrick et al., Z. Phys C65 (1995) 379.

9. S. J. Brodsky, P. Hoyer, A. H. Mueller and W-K. Tang, Nucl. Phys. B369 (1992) 519; G. Ingelman, L. Jönsson and M. Nyborg, Phys. Rev. D47 (1993) 4872. For a complete list of references see R. Vogt and S. J. Brodsky Nucl. Phys. B438 (1995) 261.

10. M. A. G. Aivazis, F. I. Olness and W-K. Tung, Phys. Rev. D50 (1994) 3085; M. A. G. Aivazis, J. C. Collins, F. I. Olness and W-K. Tung, Phys. Rev. D50 3102 (1994).

11. F. I. Olness and S. Riemersma, Phys. Rev. D51 4746 (1995).

12. M. Buza, Y. Matiounine, J. Smith, R. Migneron and W.L. van Neerven, hep$\mathrm{ph} / 9601302$.

13. J. C. Collins, D. E. Soper and G. Sterman, Nucl. Phys. B263 (1986) 37. For a review see J. C. Collins, D. E. Soper and G. Sterman in Perturbative QCD ed. A. H. Mueller ( World Scientific, 1989).

14. G. P. Lepage, J. Comp. Phys. 27 (1978) 192.

15. J. A. M. Vermaseren, FORM Version 2.2b, CAN, Amsterdam, The Netherlands, 1991.

16. G. Altarelli and G. Parisi, Nucl. Phys. B126 (1977) 298; V. N. Gribov and L. N. Lipatov,
Yad. Fiz. 15 (1972) 1218 [Sov. J. Nucl. Phys. 15 (1972) 675].

17. B.W. Harris, J. Smith and R. Vogt, Nucl. Phys. B461 (1996) 181. 